\title{
A Novel Approach to Automated Bloodstain Pattern Analysis Using an Active Bloodstain Shape Model
}

Authors: Philip Joris, Wim Develter, Els Jenar, Dirk Vandermeulen, Walter Coudyzer, Joke Wuestenbergs, Bart De Dobbelaer, Wim Van de Voorde, Eric Geusens, Peter Claes

1: University Hospitals Leuven (UZ) Belgium, Department of Forensic Medicine.
2: University Hospitals Leuven (UZ) Belgium, Department of Radiology.
3: University of Leuven (KUL) Belgium, Department of Electrical Engineering, ESAT/PSI, Medical Image
Computing \& UZ Leuven, Medical Imaging Research Center \& iMinds, Future Health Department.

Objectives - Conventional blood pattern analysis is a tedious and timeconsuming process due to the many actions that must be performed by the pattern analyst. Digital photographs can now be employed to aid in the process of scene analysis, by letting computer programs perform some of the required steps. However, current computerized methods are all based upon the assumption that any stain can be approximated by a simple ellipse, while manual work is still required.

Materials and methods - This work presents a novel approach, employing a regressed active shape model to approximate bloodstains. This Active Bloodstain Shape Model (ABSM) uses a regression to correlate the shape of a stain's approximation to its impact angle. This model is then deployed in a software pipeline, aiming to eliminate any user-input from the process. Fiducial markers are positioned in a crime scene, allowing for fully automated pattern analysis. Images are cleaned of any perspective distortions, after which stains are segmented and analyzed using said model. A robust principal component analysis (PCA) is then used to analyze the intersections of the reconstructed flight paths.

Results - Experimental results have demonstrated that the ABSM is able to better approximate stains, while accuracy on predicted impact angles is increased. Both simulated as well as real crimes scenes have confirmed the working of the automated pipeline.

Conclusion - Because the ABSM learns from experimental data, it is able to better approximate stains, resulting in an increased accuracy. The addition of the software pipeline removed the need for almost all user-input. 